\section{Associação entre realização de refeições com os pais ou responsáveis e obesidade em adolescentes brasileiros}

\author{
Association between sharing meals with parents \\ or guardians and obesity in Brazilian adolescents
}

\section{Asociación entre realización de comidas con los padres o tutores y obesidad en adolescentes brasileños}

Juliana Ilídio da Silva 1 Amanda Cristina de Souza Andrade 1 Katia Vergetti Bloch 1 Gisela Soares Brunken 1

doi: 10.1590/0102-311X00104419

\section{Resumo}

O objetivo do trabalho foi analisar a associação da frequência de almoçar e jantar com os pais/responsáveis e obesidade em adolescentes brasileiros participantes do Estudo de Riscos Cardiovasculares em Adolescentes (ERICA). Estudo seccional, de base escolar, com adolescentes de 12 a 17 anos. A obesidade foi classificada pelo índice de massa corporal baseado nos critérios da Organização Mundial da Saúde, segundo idade e sexo. A associação entre obesidade e almoço e jantar com os pais/responsáveis (nunca, às vezes, quase todos os dias e todos os dias) foi investigada pela razão de prevalência bruta e ajustada para estratos de sexo e faixa etária. Foram avaliados 71.740 adolescentes. Desses, almoçavam e jantavam com os pais/responsáveis todos os dias ou quase todos os dias, respectivamente, cerca de $48 \%$ e $60 \%$ das meninas e $56 \%$ e $65 \%$ dos meninos. Os meninos que almoçavam e jantavam com os pais/ responsáveis quase todos os dias e todos os dias apresentaram menor prevalência de obesidade. Na estratificação por faixa etária, apenas os meninos mais novos que afirmaram almoçar com os pais/responsáveis às vezes $(R P=0,64$; IC95\%: 0,46-0,89), quase todos os dias $(R P=0,50$; IC95\%: 0,37-0,69) e todos os dias $(R P=0,65$; IC95\%: 0,49-0,85) e jantar com os pais/responsáveis todos os dias $(R P=0,61$; IC95\%: 0,43-0,87) apresentaram menor prevalência de obesidade. Os achados reforçam a importância da promoção de comportamentos alimentares saudáveis no ambiente familiar como parte das estratégias de prevenção da obesidade em adolescentes.

Adolescente; Obesidade; Índice de Massa Corporal; Refeições; Inquéritos Epidemiológicos

\section{Correspondência}

J. I. Silva

Instituto de Estudos em Saúde Coletiva, Universidade Federal de Mato Grosso.

Av. Edgar Vieira 1608, Cuiabá, MT 78068-401, Brasil.

julianailidio_enf@hotmail.com

1 Instituto de Saúde Coletiva, Universidade Federal de Mato Grosso, Cuiabá, Brasil. 


\section{Introdução}

A obesidade é uma doença complexa, multifatorial e um importante problema de saúde pública no Brasil e no mundo, em todas as faixas etárias e em ambos os sexos 1 . O aumento da prevalência de obesidade em adolescentes 1 tem preocupado as autoridades públicas, por estar relacionado ao elevado potencial de morbimortalidades na vida adulta com diminuição da expectativa de vida 2 . No Brasil, de 1974-1975 a 2008-2009, a prevalência de obesidade em adolescentes aumentou de 0,4\% para 5,9\% no sexo masculino, e de $0,7 \%$ para $4 \%$ no feminino 3 .

Fatores genéticos, fisiológicos, ambientais, sociais e comportamentais interagem na gênese da obesidade, figurando dentre eles o ambiente familiar. A refeição é um aspecto do ambiente familiar que está associado ao bem-estar e pode ser considerado um indicador de estilo de vida saudável em crianças, jovens e adultos ${ }^{4}$. A refeição em família inclui, entre seus benefícios para os adolescentes, proteção contra comportamentos de risco e comportamentos alimentares desordenados, melhor bem-estar psicossocial e desempenho acadêmico, mais diálogo e coesão entre pais e filhos ${ }^{5}$. O hábito de realizar refeições em família proporciona um ambiente para os pais incentivarem comportamentos saudáveis aos filhos 5 , que podem transcender ao longo da vida ${ }^{4}$. Além de oportunizar um espaço de aprendizagem sobre hábitos alimentares e benefícios nutricionais 6 . Isso permite que seus filhos possam adquirir habilidades para escolha e aquisição dos alimentos, planejamento e preparo das refeições 7,8 .

Em revisão dos principais achados de uma década de pesquisa do projeto Eating Among Teens, sobre padrões alimentares e problemas relacionados ao peso em adolescentes dos Estados Unidos, foram observadas nas análises transversais associações inversas significativas entre frequência de refeição familiar e excesso de peso em meninas mais jovens, mas não foi observado em meninos mais jovens ou adolescentes mais velhos de ambos os sexos. $\mathrm{Na}$ análise longitudinal, a frequência de refeição em família não esteve associada à obesidade em adolescentes 9 .

Embora exista evidência de que fazer refeições em família com frequência esteja associado a um menor índice de massa corporal (IMC) em crianças e adolescentes 10, uma revisão sistemática não identificou evidências consistentes de associação inversa entre compartilhar refeições em família e probabilidade de excesso de peso na infância e adolescência 11.

No Brasil, estudos que avaliaram a influência das refeições com os pais/responsáveis em relação à obesidade em adolescentes ainda são escassos. Nesse contexto, o objetivo deste trabalho foi analisar a associação entre obesidade e a frequência de almoçar e jantar com os pais ou responsáveis em adolescentes brasileiros, considerando-se o sexo e a faixa etária.

\section{Métodos}

Este trabalho usa os dados do Estudo de Riscos Cardiovasculares em Adolescentes (ERICA). Estudo seccional, de base escolar, com abrangência nacional, cujo objetivo foi estimar a prevalência de fatores de risco cardiovascular e da síndrome metabólica em adolescentes de 12 a 17 anos, de ambos os sexos, que frequentavam escolas das cidades brasileiras com mais de 100 mil habitantes 12 . A coleta de dados foi conduzida entre 2013 e 2014.

A amostra do ERICA foi dividida em 32 estratos geográficos, compostos por 26 capitais, o Distrito Federal e mais cinco conjuntos compreendendo os demais municípios com mais de 100 mil habitantes (médio e grande portes) de cada uma das cinco macrorregiões do país: Norte, Nordeste, Centro-oeste, Sudeste e Sul. Foi adotado um delineamento por conglomerado em três estágios: seleção das escolas; combinações de ano e turno; seleção da turma. A descrição detalhada do processo de amostragem pode ser obtida em publicação anterior 12 . A amostra prevista foi de 102.327 adolescentes, entretanto, 23,7\% (24.284) foram perdas devido à não resposta de nenhum bloco do questionário, recordatório alimentar de 24 horas (R24h), ou aferição de medidas antropométricas e pressão arterial. Entre os não participantes o percentual do sexo masculino, de faixa etária de 15 a 17 anos e de alunos de escolas públicas foi um pouco maior do que entre os participantes. No entanto, foram utilizados procedimentos de análise que, além de levarem em consideração o desenho de amostra complexa, usaram pesos calibrados que corrigem as estimativas de prevalência de acordo com a distribuição de idade e sexo em 
cada estrato da amostra. Essas estimativas pontuais consideram que os participantes representam os que não participaram 13. Dos 78.043 adolescentes pesquisados no ERICA, foram incluídos no presente estudo 71.740 que completaram integralmente as seguintes etapas: tiveram os dados antropométricos mensurados, responderam ao R24h e preencheram o questionário estruturado autopreenchível 13.

Os critérios de inclusão foram: adolescentes sem qualquer deficiência temporária ou definitiva, não grávidas e que cursavam o sétimo, oitavo e nono anos do Ensino Fundamental ou o primeiro, segundo ou terceiro ano do Ensino Médio em escolas públicas ou privadas nas turmas da manhã ou da tarde 12 .

As informações do questionário autopreenchível foram coletadas em sala de aula, utilizando-se coletor eletrônico de dados (personnal digital assistant - PDA), sob a supervisão da equipe do estudo e contendo cerca de 105 questões distribuídas em 11 blocos temáticos, abordando aspectos sociodemográficos, de saúde e estilo de vida 14.

Foram aferidas medidas de estatura e peso. $\mathrm{O}$ peso foi avaliado por uma única medida em balança eletrônica da marca Líder, modelo p150m (São Paulo, Brasil), com capacidade de 200 quilogramas e variação de 50 gramas. A estatura foi obtida pela média de duas aferições realizadas sequencialmente, utilizando-se estadiômetro portátil calibrado e desmontável, da marca Alturexata (Minas Gerais, Brasil), com resolução de milímetro e campo de uso de até 213 centímetros. Para a coleta, os adolescentes ficavam em posição ortostática, vestindo roupas leves e sem sapatos 14.

O IMC foi calculado pela razão entre o peso em kg e o quadrado da estatura em metros e classificado, baseado nos critérios da Organização Mundial da Saúde 15, segundo sexo e idade: "muito baixo peso", adolescentes com escore $-Z<-3$; "baixo peso" aqueles com escore $-Z \geq-3$ e $<-2$; "peso adequado" com escore $-Z \geq-2$ e $\leq+1$; "sobrepeso" com escore $-Z>+1$ e $\leq+2$; "obeso" com escore $Z>+2$. A variável de desfecho de estudo foi a obesidade presente (adolescentes classificados em obesos) e ausente (peso adequado e sobrepeso). Foram excluídos da análise $2.113(<3 \%)$ adolescentes de baixo peso e muito baixo peso (escore $-Z<-2$ ).

As refeições - almoçar e jantar - com os pais ou responsáveis foram as variáveis de exposição principais e medidas pelas perguntas: "Seu pai (ou padrasto) ou sua mãe (ou madrasta) ou responsável almoça com você?" e "Seu pai (ou padrasto) ou sua mãe (ou madrasta) ou responsável janta com você?", com as seguintes opções de resposta: "nunca ou quase nunca", "às vezes", "quase todos os dias" e "todos os dias".

As covariáveis avaliadas no estudo foram: sexo (masculino e feminino); idade por faixa etária (12-14 e 15-17 anos); cor da pele declarada (branca, preta, parda, amarela/indígena); macrorregião geográfica (Norte, Nordeste, Sul, Sudeste e Centro-oeste); escolaridade da mãe em anos (de 0-8, de 9-11, $\geq 12$, não sabe/não lembra/não respondeu); tipo de escola (pública ou privada); composição familiar (mora com pai e mãe, mora com pelo menos um dos pais e não mora com nenhum dos pais) foi obtida pela combinação das variáveis "morar com a mãe (sim/não)" e "morar com o pai (sim/não)"; a prática de atividade física, classificada em: inativo $(0 \mathrm{~min} / \mathrm{sem})$, insuficientemente ativo $(<300 \mathrm{~min} /$ sem) e ativo ( $\geq 300 \mathrm{~min} / \mathrm{sem}) 16$.

Um escore de bens e serviços (EBS), baseado na metodologia proposta por Levy et al. 17, também foi utilizado. Compuseram o escore os seguintes itens: presença de empregada doméstica, presença de banheiro em casa, posse de televisão, automóvel/motocicleta, máquina de lavar, videocassetes/ aparelhos de DVD, geladeira, computador. O escore foi obtido pela soma de cada item multiplicado pelo inverso da frequência de presença no total da amostra estudada. O escore foi categorizado em tercis: 1 - baixo, 2 - médio, 3 - alto.

O consumo de fibra foi estimado pela aplicação do R24h desenvolvido especificamente para o ERICA, e mensura o consumo alimentar das 24 horas antecedentes à entrevista utilizando-se um software específico (ERICA-REC24h) para o registro direto das informações em netbooks. A técnica de entrevista usada para a aplicação do R24h foi o multiple pass method 18 . Foram utilizadas fotografias incluídas no software, para estimar o tamanho da porção consumida 14. O "consumo adequado" de fibras foi estimado segundo o ponto de corte do U. S. Institute of Medicine 19, que recomenda: consumir no mínimo as seguintes quantidades, segundo sexo e faixa etária: meninos: 31g/dia ( $9-13$ anos) e $38 \mathrm{~g} / \mathrm{dia}$ (14-18 anos); e meninas $26 \mathrm{~g} / \mathrm{dia}$ (9-18 anos). O consumo de fibra é um indicador usado em estudos prévios como marcador de alimentação saudável 20,21. O baixo consumo de fibra representa baixa quantidade de frutas, vegetais e grãos integrais consumidos e está associado inversamente à 
adiposidade visceral, aos múltiplos biomarcadores de inflamação relacionados à obesidade 20 e ao desenvolvimento da obesidade geral e abdominal 21.

Todas as análises foram realizadas com o pacote estatístico Statistics/Data Analysis (Stata https://www.stata.com) versão 14.0, utilizando-se o módulo survey para análise de dados de amostra complexa. As análises foram estratificadas por sexo. A análise descritiva envolveu o cálculo da frequência relativa com os intervalos de 95\% de confiança (IC95\%). Foram estimadas prevalências e respectivos IC95\% da obesidade segundo variáveis de exposição e covariáveis, usando-se para comparar as proporções o teste de qui-quadrado (Rao-Scott).

A associação entre variáveis de exposição e o desfecho foi analisada ajustando-se o modelo de regressão de Poisson. Foram estimadas razões de prevalências brutas e ajustadas para cada estrato de sexo e de faixa etária. As variáveis com valor de $\mathrm{p}<0,20$ na análise bivariada foram usadas como variáveis de ajuste na análise multivariada quando modificavam a razão de prevalência (RP) em pelo menos 20\% (faixa etária, cor da pele, região geográfica, tipo de escola, consumo de fibras e EBS). A variável composição familiar foi mantida como ajuste nos modelos multivariados devido à sua associação com as variáveis de exposição. A variável "escolaridade da mãe” não foi utilizada na análise multivariada devido ao alto percentual de não resposta. Em todas as análises de associação com as variáveis de exposição a categoria "nunca ou quase nunca" foi a de referência. Foi considerado como significância estatística um valor de $\mathrm{p}<0,05$.

Participaram do estudo os adolescentes que assinaram o Termo de Assentimento. Foi solicitado, em cinco estados, o termo de consentimento livre e esclarecido assinado pelos pais ou responsáveis por determinação dos Comitês de Ética em Pesquisa (CEP) ou Secretaria Estadual de Educação. O ERICA foi aprovado pelo CEP de cada uma das 27 Unidades da Federação.

\section{Resultados}

No total, 71.740 adolescentes foram avaliados. A média de idades foi de 14 anos, com pouco mais da metade dos alunos na faixa etária de 12-14 anos em ambos os sexos. Os adolescentes de ambos os sexos que se autodeclararam de cor da pele parda e branca e da região Sudeste compõem a grande maioria da amostra. Um pouco mais de um quarto dos meninos e das meninas não sabia, não lembrava ou não respondeu sobre a escolaridade da mãe. Tanto no sexo masculino como no feminino, pouco mais da metade morava com pai e mãe e quase 50\% apresentaram escore de bens e serviço (ESB) médio. Em relação ao tipo de escola, pelo menos 3/4 dos meninos e das meninas frequentavam escolas públicas. O percentual de adequação de consumo de fibra foi maior para as meninas do que para os meninos. Por outro lado, os adolescentes do sexo masculino estavam mais ativos do que as do sexo feminino. Quanto às refeições com os pais/responsáveis, aproximadamente metade das meninas e dos meninos as realizavam com maior regularidade. Maior proporção de meninos referia almoçar ou jantar com os pais ou responsáveis "todos os dias". Por sua vez, as meninas apresentaram maior porcentual de "nunca ou quase nunca” almoçar ou jantar com os pais/responsáveis. A prevalência de adolescentes com obesidade foi maior nos meninos do que nas meninas (Tabela 1).

Para as análises bivariada e multivariada foram avaliados 69.627 adolescentes. A prevalência de obesidade em meninos esteve associada com quase todas as variáveis estudadas, exceto composição familiar, atividade física e jantar com os pais/responsáveis. Com relação às adolescentes, a prevalência de obesidade esteve associada apenas com faixa etária, cor da pele, região geográfica e consumo de fibra (Tabela 2).

Nos adolescentes do sexo masculino, a faixa etária modificou a associação entre almoçar e jantar com os pais/responsáveis com obesidade (Tabelas 3 e 4). As medidas de razão de prevalência brutas e ajustadas da Tabela 3 mostram para cada sexo separadamente a associação da frequência de almoçar com os pais/responsáveis com a obesidade, estratificada por faixa etária. Nos resultados ajustados, almoçar com os pais/responsáveis não apresentou associação com obesidade no sexo feminino. Mas esse hábito foi inversamente associado com obesidade nos meninos que almoçavam "às vezes", "quase todos os dias" ou "todos os dias". Com relação à faixa etária, observou-se menor prevalência de obesidade apenas nos meninos de 12-14 anos, que almoçavam "às vezes", "quase todos os dias" e "todos os dias" (Tabela 3). 
Tabela 1

Distribuição da amostra ( $\mathrm{n}=71.740$ ) por características demográficas, socioeconômicas e comportamentais, segundo sexo. Estudo de Riscos Cardiovasculares em Adolescentes (ERICA), Brasil, 2013-2014.

\begin{tabular}{|c|c|c|c|c|}
\hline \multirow[t]{2}{*}{ Variáveis } & \multicolumn{2}{|c|}{ Feminino } & \multicolumn{2}{|c|}{ Masculino } \\
\hline & $\%$ & IC95\% & $\%$ & IC95\% a \\
\hline Sexo & 49,8 & - & 50,2 & - \\
\hline \multicolumn{5}{|l|}{ Faixa etária (anos) } \\
\hline $12-14$ & 52,5 & - & 52,9 & - \\
\hline $15-17$ & 47,5 & - & 47,1 & - \\
\hline \multicolumn{5}{|l|}{ Cor da pele } \\
\hline Branca & 38,9 & 37,$1 ; 40,8$ & 39,2 & 37,$4 ; 40,9$ \\
\hline Preta & 6,6 & 6,$1 ; 7,3$ & 9,5 & 8,$5 ; 10,6$ \\
\hline Parda & 50,3 & 48,$7 ; 51,9$ & 44,8 & 43,$2 ; 46,4$ \\
\hline Amarela & 2,2 & 1,$9 ; 2,5$ & 2,0 & 1,$7 ; 2,3$ \\
\hline Indígena & 0,4 & 0,$3 ; 0,5$ & 0,9 & 0,$7 ; 1,2$ \\
\hline NS/NR & 1,5 & 1,$3 ; 1,8$ & 3,7 & 3,$4 ; 4,2$ \\
\hline \multicolumn{5}{|l|}{ Região geográfica } \\
\hline Norte & 8,5 & 8,$5 ; 8,5$ & 8,4 & 8,$4 ; 8,4$ \\
\hline Nordeste & 21,4 & 21,$4 ; 21,4$ & 21,2 & 21,$2 ; 21,2$ \\
\hline Centro-oeste & 7,7 & 7,$7 ; 7,7$ & 7,6 & 7,$6 ; 7,6$ \\
\hline Sudeste & 50,6 & 50,$6 ; 50,6$ & 50,9 & 50,$9 ; 50,9$ \\
\hline Sul & 11,8 & 11,$8 ; 11,8$ & 11,8 & 11,$8 ; 11,8$ \\
\hline \multicolumn{5}{|l|}{ Escolaridade da mãe (anos) } \\
\hline $0-8$ & 27,5 & 25,$6 ; 29,5$ & 25,4 & 23,$2 ; 27,7$ \\
\hline $9-11$ & 26 & 24,$6 ; 27,5$ & 23,6 & 22,$2 ; 25,1$ \\
\hline$\geq 12$ & 19,1 & 17,$3 ; 21,1$ & 22,6 & 20,$4 ; 25,0$ \\
\hline NS/NL/NR & 27,4 & 26,$2 ; 28,6$ & 28,4 & 27,$0 ; 29,8$ \\
\hline \multicolumn{5}{|l|}{ Tipo de escola } \\
\hline Pública & 83,2 & 78,$7 ; 87,0$ & 82,0 & 77,$2 ; 86,0$ \\
\hline Privada & 16,8 & 13,$0 ; 21,3$ & 18,0 & 14,$0 ; 22,8$ \\
\hline \multicolumn{5}{|l|}{ Composição familiar } \\
\hline Mora com pai e mãe & 55,1 & 53,$8 ; 56,5$ & 59,4 & 57,$4 ; 61,3$ \\
\hline Mora com pelo menos um dos pais & 38,8 & 37,$6 ; 40,0$ & 34,9 & 32,$9 ; 37,0$ \\
\hline Não mora com nenhum dos pais & 6,0 & 5,$5 ; 6,6$ & 5,7 & 5,$0 ; 6,6$ \\
\hline \multicolumn{5}{|l|}{ Escore de bens e serviço } \\
\hline Baixo & 37,5 & 35,$7 ; 39,4$ & 32,8 & 30,$8 ; 34,9$ \\
\hline Médio & 47,0 & 45,$3 ; 48,6$ & 48,4 & 46,$6 ; 50,2$ \\
\hline Alto & 15,5 & 14,$1 ; 17,0$ & 18,8 & 17,$4 ; 20,4$ \\
\hline \multicolumn{5}{|l|}{ Consumo de fibra } \\
\hline Adequado & 15,2 & 13,$7 ; 16,7$ & 11,0 & 9,$4 ; 12,9$ \\
\hline Não Adequado & 84,8 & 83,$3 ; 86,3$ & 89,0 & 87,$1 ; 90,6$ \\
\hline \multicolumn{5}{|l|}{ Atividade Física } \\
\hline Inativo & 39,8 & 38,$7 ; 40,9$ & 13,4 & 12,$4 ; 14,5$ \\
\hline Insuficientemente ativo & 30,7 & 29,$4 ; 32,0$ & 24,7 & 23,$6 ; 25,8$ \\
\hline Ativo & 29,5 & 28,$3 ; 30,7$ & 62,0 & 60,$6 ; 63,4$ \\
\hline \multicolumn{5}{|l|}{ Almoçar com os pais/responsáveis } \\
\hline Nunca ou quase nunca & 16,8 & 15,$9 ; 17,7$ & 13,5 & 12,$5 ; 14,5$ \\
\hline Às vezes & 35,6 & 34,$4 ; 36,8$ & 30,9 & 29,$7 ; 32,2$ \\
\hline Quase todos os dias & 19,8 & 18,$8 ; 21,0$ & 21,2 & 19,$9 ; 22,6$ \\
\hline Todos os dias & 27,8 & 26,$7 ; 28,9$ & 34,4 & 33,$3 ; 35,5$ \\
\hline
\end{tabular}

(continua) 
Tabela 1 (continuação)

\begin{tabular}{lcccc}
\hline Variáveis & \multicolumn{2}{c}{ Feminino } & \multicolumn{2}{c}{ Masculino } \\
& $\%$ & IC95\% & $\%$ & IC95\% a \\
\hline Jantar com os pais/responsáveis & & & & \\
$\quad$ Nunca ou quase nunca & 12,0 & 11,$3 ; 12,7$ & 10,3 & 9,$4 ; 11,2$ \\
Às vezes & 27,9 & 26,$8 ; 29,1$ & 24,4 & 23,$4 ; 25,5$ \\
$\quad$ Quase todos os dias & 20,5 & 19,$6 ; 21,4$ & 21,1 & 20,$2 ; 22,1$ \\
$\quad$ Todos os dias & 39,6 & 38,$4 ; 40,8$ & 44,2 & 42,$9 ; 45,4$ \\
Classificação do IMC & & & & \\
$\quad$ Muito baixo peso & 0,2 & 0,$1 ; 0,3$ & 0,7 & 0,$5 ; 1,0$ \\
$\quad$ Baixo peso & 2,0 & 1,$7 ; 2,3$ & 2,8 & 2,$4 ; 3,2$ \\
Adequado & 72,7 & 71,$1 ; 74,2$ & 70,8 & 69,$3 ; 72,3$ \\
Sobrepeso & 17,5 & 16,$3 ; 18,8$ & 16,6 & 15,$5 ; 17,7$ \\
Obesidade & 7,6 & 7,$0 ; 8,2$ & 9,2 & 8,$4 ; 9,9$ \\
\hline
\end{tabular}

IC95\%: intervalo de 95\% de confiança; IMC: índice de massa corporal; NL: não lembra; NR: não respondeu; NS: não sabe.

\section{Tabela 2}

Prevalências ( $n=69.627$ ) e intervalos de 95\% de confiança (IC95\%) de obesidade por sexo, segundo variáveis demográficas, socioeconômicas e comportamentais. Estudo de Riscos Cardiovasculares em Adolescentes (ERICA), Brasil, 2013-2014.

\begin{tabular}{|c|c|c|c|c|c|c|}
\hline \multirow[t]{2}{*}{ Variáveis } & \multicolumn{3}{|c|}{ Feminino } & \multicolumn{3}{|c|}{ Masculino } \\
\hline & $\%$ & IC95\% & Valor de $p$ & $\%$ & IC95\% & Valor de $p$ \\
\hline \multicolumn{7}{|l|}{ Faixa etária (anos) } \\
\hline $12-14$ & 8,7 & 7,$9 ; 9,7$ & & 11,2 & 10,$1 ; 12,4$ & \\
\hline $15-17$ & 6,8 & 5,$8 ; 7,8$ & 0,008 & 7,6 & 6,$8 ; 8,4$ & $<0,001$ \\
\hline \multicolumn{7}{|l|}{ Cor da pele } \\
\hline Branca & 8,8 & 7,$6 ; 10,0$ & & 11,9 & 10,$7 ; 13,2$ & \\
\hline Preta & 8,9 & 7,$0 ; 11,2$ & & 7,6 & 6,$0 ; 9,6$ & \\
\hline Parda & 6,7 & 6,$0 ; 7,6$ & & 7,4 & 6,$4 ; 8,5$ & \\
\hline Amarela e indígena & 7,4 & 5,$0 ; 10,8$ & 0,005 & 10,4 & 6,$4 ; 16,4$ & $<0,001$ \\
\hline \multicolumn{7}{|l|}{ Região geográfica } \\
\hline Norte & 5,7 & 5,$0 ; 6,6$ & & 7,8 & 6,$7 ; 9,0$ & \\
\hline Nordeste & 7,0 & 6,$2 ; 7,9$ & & 8,7 & 7,$2 ; 10,6$ & \\
\hline Centro-oeste & 6,0 & 5,$4 ; 6,7$ & & 9,2 & 7,$6 ; 11,0$ & \\
\hline Sudeste & 8,2 & 7,$2 ; 9,4$ & & 9,3 & 8,$2 ; 10,6$ & \\
\hline Sul & 10,2 & 8,$2 ; 12,5$ & $<0,001$ & 12,9 & 11,$4 ; 14,5$ & $<0,003$ \\
\hline \multicolumn{7}{|l|}{ Escolaridade da mãe (anos) } \\
\hline $0-8$ & 8,9 & 7,$4 ; 10,7$ & & 7,6 & 6,$3 ; 9,3$ & \\
\hline $9-11$ & 8,3 & 7,$1 ; 9,7$ & & 10,5 & 9,$2 ; 12,0$ & \\
\hline$\geq 12$ & 7,4 & 6,$2 ; 8,9$ & 0,326 & 11,0 & 9,$5 ; 12,7$ & 0,003 \\
\hline \multicolumn{7}{|l|}{ Tipo de escola } \\
\hline Pública & 7,6 & 6,$9 ; 8,3$ & & 8,7 & 7,$9 ; 9,5$ & \\
\hline Privada & 9,0 & 7,$7 ; 10,6$ & 0,051 & 13,2 & 11,$6 ; 15,0$ & $<0,001$ \\
\hline \multicolumn{7}{|l|}{ Composição familiar } \\
\hline Mora com pai e mãe & 8,2 & 7,$2 ; 9,2$ & & 9,5 & 8,$4 ; 10,7$ & \\
\hline Mora com pelo menos um dos pais & 7,4 & 6,$3 ; 8,7$ & & 9,2 & 7,$9 ; 10,8$ & \\
\hline Não mora com nenhum dos pais & 6,6 & 4,$9 ; 8,8$ & 0,378 & 11,2 & 8,$0 ; 15,4$ & 0,619 \\
\hline
\end{tabular}

(continua) 
Tabela 2 (continuação)

\begin{tabular}{|c|c|c|c|c|c|c|}
\hline \multirow[t]{2}{*}{ Variáveis } & \multicolumn{3}{|c|}{ Feminino } & \multicolumn{3}{|c|}{ Masculino } \\
\hline & $\%$ & IC95\% & Valor de $\mathrm{p}$ & $\%$ & IC95\% & Valor de $p$ \\
\hline \multicolumn{7}{|l|}{ Escore de bens e serviço } \\
\hline Baixo & 7,3 & 6,$2 ; 8,6$ & & 8,4 & 7,$4 ; 9,4$ & \\
\hline Médio & 8,3 & 7,$2 ; 9,5$ & & 9,5 & 8,$5 ; 10,6$ & \\
\hline Alto & 7,6 & 6,$1 ; 9,5$ & 0,521 & 11,7 & 9,$9 ; 13,8$ & 0,004 \\
\hline \multicolumn{7}{|l|}{ Consumo de fibra } \\
\hline Adequado & 4,7 & 3,$6 ; 6,3$ & & 6,2 & 4,$7 ; 8,2$ & \\
\hline Não adequado & 8,3 & 7,$7 ; 9,1$ & $<0,001$ & 9,9 & 9,$1 ; 10,8$ & $<0,002$ \\
\hline \multicolumn{7}{|l|}{ Atividade física } \\
\hline Inativo & 6,9 & 6,$1 ; 7,8$ & & 10,2 & 8,$6 ; 12,0$ & \\
\hline Insuficientemente ativo & 8,3 & 7,$0 ; 10,0$ & & 9,9 & 8,$5 ; 11,4$ & \\
\hline Ativo & 8,4 & 7,$3 ; 9,6$ & 0,138 & 9,2 & 8,$3 ; 10,2$ & 0,444 \\
\hline \multicolumn{7}{|c|}{ Almoçar com os pais/responsáveis } \\
\hline Nunca ou quase nunca & 8,3 & 6,$8 ; 10,0$ & & 11,9 & 9,$7 ; 14,5$ & \\
\hline Às vezes & 7,0 & 6,$0 ; 8,1$ & & 8,4 & 7,$4 ; 9,6$ & \\
\hline Quase todos os dias & 7,8 & 6,$4 ; 9,4$ & & 7,5 & 6,$2 ; 9,2$ & \\
\hline Todos os dias & 8,6 & 7,$2 ; 10,2$ & 0,289 & 10,7 & 9,$2 ; 12,4$ & $<0,002$ \\
\hline \multicolumn{7}{|c|}{ Jantar com os pais/responsáveis } \\
\hline Nunca ou quase nunca & 8,2 & 6,$6 ; 10,1$ & & 11,2 & 8,$7 ; 14,4$ & \\
\hline Às vezes & 6,8 & 5,$7 ; 8,1$ & & 9,5 & 8,$31 ; 10,8$ & \\
\hline Quase todos os dias & 7,9 & 6,$5 ; 9,6$ & & 8,5 & 7,$1 ; 10,2$ & \\
\hline Todos os dias & 8,3 & 7,$4 ; 9,3$ & 0,290 & 9,5 & 8,$4 ; 10,8$ & 0,333 \\
\hline
\end{tabular}

\section{Tabela 3}

Razões de prevalências (RP) e intervalos de 95\% de confiança (IC95\%) de obesidade com frequência de almoçar com os pais ou responsáveis, segundo faixa etária, em adolescentes por sexo. Estudo de Riscos Cardiovasculares em Adolescentes (ERICA), Brasil, 2013-2014.

\begin{tabular}{|c|c|c|c|c|}
\hline \multirow{2}{*}{$\begin{array}{l}\text { Almoçar com os pais/ } \\
\text { responsáveis }\end{array}$} & \multicolumn{2}{|c|}{ Feminino } & \multicolumn{2}{|c|}{ Masculino } \\
\hline & $\mathrm{RP}_{\text {bruta }}$ (IC95\%) & $\mathbf{R P}_{\text {ajustada }}$ (IC95\%) & $\mathrm{RP}_{\text {bruta }}(\mathrm{IC95 \% )}$ & $\mathrm{RP}_{\text {ajustada }}(\mathrm{IC} 95 \%)$ \\
\hline \multicolumn{5}{|l|}{ Geral * } \\
\hline Nunca & 1,00 & 1,00 & 1,00 & 1,00 \\
\hline Às vezes & $0,84(0,66 ; 1,08)$ & $0,82(0,64 ; 1,04)$ & $0,71(0,55 ; 0,91)$ & $0,68(0,53 ; 0,88)$ ** \\
\hline Quase todos os dias & $0,94(0,71 ; 1,25)$ & $0,90(0,69 ; 1,17)$ & $0,63(0,48 ; 0,83)$ & $0,59(0,45 ; 0,77)$ ** \\
\hline Todos os dias & $1,04(0,80 ; 1,36)$ & $0,95(0,74 ; 1,21)$ & $0,90(0,70 ; 1,15)$ & $0,76(0,60 ; 0,96)$ *** \\
\hline \multicolumn{5}{|l|}{ Faixa etária (anos) \# } \\
\hline \multicolumn{5}{|l|}{$12-14$} \\
\hline Nunca & 1,00 & 1,00 & 1,00 & 1,00 \\
\hline Às vezes & $0,89(0,65 ; 1,23)$ & $0,88(0,64 ; 1,21)$ & $0,67(0,48 ; 0,93)$ & $0,64(0,46 ; 0,89) * \star$ \\
\hline Quase todos os dias & $1,03(0,71 ; 1,51)$ & $1,05(0,71 ; 1,56)$ & $0,54(0,39 ; 0,73)$ & $0,50(0,37 ; 0,69) * \star$ \\
\hline Todos os dias & $1,15(0,81 ; 1,64)$ & $1,06(0,75 ; 1,51)$ & $0,70(0,52 ; 0,96)$ & $0,65(0,49 ; 0,85) * *$ \\
\hline \multicolumn{5}{|l|}{$15-17$} \\
\hline Nunca & 1,00 & 1,00 & 1,00 & 1,00 \\
\hline Às vezes & $0,79(0,55 ; 1,13)$ & $0,78(0,55 ; 1,10)$ & $0,72(0,50 ; 1,04)$ & $0,72(0,50 ; 1,02)$ \\
\hline Quase todos os dias & $0,81(0,52 ; 1,25)$ & $0,76(0,51 ; 1,14)$ & $0,71(0,46 ; 1,11)$ & $0,73(0,48 ; 1,11)$ \\
\hline Todos os dias & $0,84(0,58 ; 1,23)$ & $0,84(0,59 ; 1,18)$ & $1,08(0,74 ; 1,58)$ & $0,98(0,68 ; 1,41)$ \\
\hline
\end{tabular}

* Ajustado por faixa etária, cor da pele, tipo de escola, região geográfica, consumo de fibra (g/dia), escore de bens e serviços e composição familiar;

** Valor de $p<0,01$;

*** Valor de $p<0,05$;

\# Ajustado por cor da pele, região geográfica, tipo de escola, consumo de fibra (g/dia) e escore de bens e serviços e composição familiar. 
Em relação a jantar com pais/responsáveis, as razões de prevalências ajustadas mostram não haver associação com obesidade no sexo feminino; já os meninos que jantavam "quase todos os dias" e "todos os dias" estiveram mais protegidos da obesidade. Em relação à estratificação por faixa etária, jantar com os pais/responsáveis foi associado à obesidade apenas nos meninos de 12-14 anos, em que os que referiram jantar "todos os dias" apresentaram menor prevalência de obesidade (Tabela 4).

\section{Discussão}

Os resultados mostraram que pelo menos metade dos adolescentes realizava refeições com os pais/ responsáveis quase todos os dias ou todos os dias, sendo que os meninos apresentaram maior frequência de refeições com os pais/responsáveis do que as meninas. Para os adolescentes brasileiros, entre os meninos houve menor prevalência de obesidade nos que relataram almoçar e jantar com os pais ou responsáveis, já entre as meninas não foi observada associação significativa. Foi observada modificação de efeito na associação de almoçar e jantar com os pais/responsáveis e obesidade para meninos de 12-14 anos.

Dados de escolares participantes de outro inquérito brasileiro, a Pesquisa Nacional de Saúde do Escolar (PeNSE), apresentou porcentual de refeições (almoçar ou jantar) com os pais um pouco mais alto; em 2015, 74\% dos estudantes no $9^{\circ}$ ano do Ensino Fundamental informaram ter este hábito, no mínimo, em cinco dias da semana que antecederam a entrevista 22. Uma possível explicação para a diferença observada pode ser o fato de a PeNSE de 2015 avaliar a presença dos pais/responsáveis durante as refeições apenas em estudantes do $9^{\circ}$ ano do Ensino Fundamental. O ERICA avaliou alunos do $7^{\circ}$ ano do Ensino Fundamental ao $3^{\circ}$ ano do Ensino Médio. Dessa forma, os adolescentes do ERICA são mais velhos e com o aumento da idade os jovens tendem a participar menos da vida familiar.

\section{Tabela 4}

Razões de prevalências (RP) e intervalos de 95\% de confiança (IC95\%) de obesidade com frequência de jantar com os pais ou responsáveis, segundo faixa etária, em adolescentes por sexo. Estudo de Riscos Cardiovasculares em Adolescentes (ERICA), Brasil, 2013-2014.

\begin{tabular}{|c|c|c|c|c|}
\hline \multirow{2}{*}{$\begin{array}{l}\text { Jantar com os pais/ } \\
\text { responsáveis }\end{array}$} & \multicolumn{2}{|c|}{ Feminino } & \multicolumn{2}{|c|}{ Masculino } \\
\hline & $\mathrm{RP}_{\text {bruta }}$ (IC95\%) & $\mathrm{RP}_{\text {ajustada }}(\mathrm{IC} 95 \%)$ & $\mathrm{RP}_{\text {bruta }}(\mathrm{IC95 \% )}$ & $\mathrm{RP}_{\text {ajustada }}(\mathrm{IC} 95 \%)$ \\
\hline \multicolumn{5}{|l|}{ Geral * } \\
\hline Nunca & 1,00 & 1,00 & 1,00 & 1,00 \\
\hline Às vezes & $0,83(0,64 ; 1,07)$ & $0,81(0,63 ; 1,03)$ & $0,84(0,62 ; 1,15)$ & $0,84(0,62 ; 1,12)$ \\
\hline Quase todos os dias & $0,97(0,72 ; 1,31)$ & $0,90(0,67 ; 1,22)$ & $0,76(0,55 ; 1,05)$ & $0,72(0,54 ; 0,98) * *$ \\
\hline Todos os dias & $1,02(0,81 ; 1,27)$ & $0,92(0,74 ; 1,14)$ & $0,85(0,65 ; 1,11)$ & $0,74(0,56 ; 0,97)$ ** \\
\hline \multicolumn{5}{|l|}{ Faixa etária (anos) $* \star \star$} \\
\hline \multicolumn{5}{|l|}{$12-14$} \\
\hline Nunca & 1,00 & 1,00 & 1,00 & 1,00 \\
\hline Às vezes & $0,74(0,50 ; 1,08)$ & $0,72(0,52 ; 1,02)$ & $0,83(0,55 ; 1,24)$ & $0,81(0,55 ; 1,20)$ \\
\hline Quase todos os dias & $0,93(0,60 ; 1,45)$ & $0,89(0,58 ; 1,37)$ & $0,70(0,45 ; 1,08)$ & $0,69(0,46 ; 1,01)$ \\
\hline Todos os dias & $0,98(0,68 ; 1,44)$ & $0,90(0,65 ; 1,26)$ & $0,65(0,46 ; 0,92)$ & $0,61(0,43 ; 0,87)^{\#}$ \\
\hline \multicolumn{5}{|l|}{$15-17$} \\
\hline Nunca & 1,00 & 1,00 & 1,00 & 1,00 \\
\hline Às vezes & $0,92(0,63 ; 1,34)$ & $0,92(0,61 ; 1,37)$ & $0,80(0,52 ; 1,23)$ & $0,85(0,54 ; 1,31)$ \\
\hline Quase todos os dias & $0,96(0,66 ; 1,42)$ & $0,94(0,63 ; 1,41)$ & $0,78(0,50 ; 1,22)$ & $0,77(0,49 ; 1,20)$ \\
\hline Todos os dias & $0,95(0,73 ; 1,24)$ & $0,94(0,71 ; 1,25)$ & $1,07(0,69 ; 1,66)$ & $1,00(0,64 ; 1,57)$ \\
\hline
\end{tabular}

* Ajustado por faixa etária, cor da pele, tipo de escola, região geográfica, consumo de fibra g/dia, escore de bens e serviços e composição familiar;

** Valor de $\mathrm{p}<0,05$;

*** Ajustado por cor da pele, região geográfica, tipo de escola, consumo de fibra g/dia, escore de bens e serviços e composição familiar;

\# Valor de $\mathrm{p}<0,01$. 
O percentual mais expressivo de almoçar e de jantar "todos os dias" com os pais/responsáveis dentre os meninos é semelhante ao de outras pesquisas nacionais e internacionais que também observaram entre os meninos maior participação em refeições com a família 9,23. Os adolescentes do sexo feminino estavam mais predispostos aos comportamentos alimentares desordenados e preocupação com a imagem corporal 24, o que poderia contribuir para a menor frequência de refeições com os pais/responsáveis. As refeições em família permitem monitorar a mudança de padrões alimentares e identificar precocemente possíveis problemas 25 . Um estudo prévio mostrou que realizar refeições em família é fator de proteção para a ocorrência de comportamentos alimentares desordenados e insatisfação corporal 26, principalmente entre as meninas.

Para o total de estudantes deste estudo, os meninos estiveram mais protegidos da obesidade ao almoçar e jantar com os pais/responsáveis do que as meninas. Esses achados diferem de outras pesquisas que investigaram diferenças por sexos em adolescentes. Um trabalho realizado no Canadá 27 e nos Estados Unidos 28 identificou, após ajuste, associação inversa entre frequência de refeições familiares e IMC apenas nas meninas. Outros estudos, conduzidos nos Estados Unidos, mostraram que a frequência de refeições familiares foi associada inversamente ao escore $-Z$ do IMC em adolescentes de ambos os sexos 29,30. Por outro lado, análises longitudinais, também com representatividade da população americana, não observaram associações entre refeições familiares e risco de excesso de peso em ambos os sexos 31,32.

A associação encontrada somente entre os meninos poderia ser explicada pelo maior percentual de frequência de refeições com os pais/responsáveis neste grupo. Talvez por isso estiveram mais sujeitos à melhoria nos hábitos alimentares pelo contato com os pais 23,30 e maior proximidade/conexão familiar 5. Um estudo com 200 famílias mostrou que aquelas com crianças (cinco a doze anos) com peso adequado passaram mais tempo reunidas durante as refeições e se envolveram em comunicação interpessoal de maneira positiva do que as famílias com crianças obesas 33 . Outro estudo realizado nos Estados Unidos mostrou que nas famílias com maior comunicação, afeto e envolvimento interpessoal durante as refeições familiares, os adolescentes apresentaram IMC mais baixo 34 .

Os dados da PeNSE e outras análises dos dados do ERICA mostraram que os meninos apresentaram mais comportamentos de risco à saúde em comparação às meninas, com consumo mais frequente de fast food e maior consumo de refrigerantes, menor consumo de legumes ou verduras 22, maior tempo de exposição a telas ( $\geq 2$ horas) ${ }^{35}$, assim como maior ingestão média de energia e de sódio ${ }^{36}$. Essas condições podem alterar o equilíbrio entre o consumo e o gasto de energia, contribuindo para um maior risco de obesidade 6 . Os resultados desse estudo sugerem que os meninos tendem a se beneficiar com o hábito de fazer refeições com a família, o que pode contribuir para a diminuição desses comportamentos de risco nesse grupo.

A associação inversa entre a frequência de almoçar e jantar com os pais/responsáveis e obesidade entre os meninos mais novos (12-14 anos) difere dos achados de Taveras et al. 31. Esses autores não observaram associação entre jantar em família e excesso de peso ao estratificar por sexo e faixa etária (9-12 anos; $\geq 13$ anos). Já a coorte realizada em Minnesota, Estados Unidos, com adolescentes com média de idade de 15 anos, observou associação entre frequência de refeições familiares e excesso de peso apenas em meninas mais novas (Ensino Fundamental) 37.

Os resultados indicaram que os meninos mais novos que faziam refeições com os pais/responsáveis tinham prevalências de obesidade menores do que às de meninos que nunca faziam. Um estudo de revisão apontou que a idade foi um dos fatores associados à frequência de refeições em família entre crianças e adolescentes 38 . Um trabalho realizado no Brasil mostrou maior prevalência de consumo de refeições com os pais ou responsáveis entre os adolescentes mais novos (12-14 anos) 39. A frequência das refeições não foi associada à prevalência de obesidade nos adolescentes mais velhos, $\mathrm{o}$ que poderia ser explicado pelo aumento da autonomia em relação aos comportamentos alimentares, com o aumento da idade 40.

Um estudo realizado com dados do ERICA mostrou que a frequência de refeições na companhia dos pais/responsáveis foi diferente entre as regiões geográficas do Brasil e tipo de escola, sendo maior entre estudantes da Região Sul e da rede privada 39. Isso reflete, provavelmente, diferenças nas características do ambiente familiar e das refeições, como o tamanho das porções e qualidade dos alimentos consumidos, relacionamento interpessoal familiar, planejamento das refeições, refeições assistindo à 
TV, escolaridade dos pais, renda da família, estrutura familiar, restrições de tempo e situação ocupacional dos pais $4,5,38$.

Com o objetivo de garantir a promoção da saúde a um maior número de crianças e adolescentes, foi implantado em 1955 no Brasil o Programa Nacional de Alimentação Escolar (PNAE). Atualmente, o Programa prevê a oferta de merenda escolar e ações de educação alimentar e nutricional a estudantes de toda a educação básica matriculados em escolas públicas, filantrópicas e em entidades comunitárias 41. O Programa não cobre alunos de escolas privadas. Dessa forma, a refeição escolar oferecida no ensino público, associada à menor proporção de vendas e divulgação de alimentos processados e ultraprocessados nas dependências das escolas, pode proporcionar um ambiente alimentar mais saudável. Já os alunos das escolas privadas estão mais expostos à predominância de ambiente alimentar obesogênico ${ }^{42}$. Embora não tenha sido avaliado, sugere-se que a maior exposição dos adolescentes do ensino privado aos ambientes obesogênicos nas dependências das escolas pode tornar as refeições com os pais/responsáveis estratégias importantes para a prevenção da obesidade.

As refeições em família oferecem oportunidades para a promoção à saúde dos adolescentes com potencial para propiciar benefícios em longo prazo. Na adolescência, as refeições em família não contribuem apenas na prevenção da obesidade, mas também podem moldar os hábitos alimentares (maior consumo de alimentos saudáveis e menor de alimentos não saudáveis) 9,23, reduzir os comportamentos de riscos e problemas de saúde mental 26. Também promovem outros fatores psicossociais, incluindo apoio familiar percebido, vínculo familiar, convívio social, transmissão de valores, melhor desenvolvimento cognitivo e habilidades de comunicação 5 . Em longo prazo, quando as refeições em família são estabelecidas na adolescência, podem melhorar a comunicação entre pais e filhos 43 , influenciar positivamente a qualidade da alimentação e os padrões de refeição 44 e diminuir as chances de excesso de peso 45 na fase adulta. Esses resultados reforçam a importância de promover as refeições em família.

As limitações deste estudo incluem o desenho seccional, que dificulta a determinação de uma relação de causa-efeito por não permitir estabelecer a relação cronológica entre exposição e desfecho. O uso de dados autorreferidos pode apresentar viés de informação, mas os possíveis erros de classificação seriam não diferenciais, levando à subestimação de efeitos estimados. Os adolescentes de baixo peso e muito baixo peso foram excluídos da análise por formarem um grupo muito específico, com predisposição a apresentarem deficiências nutricionais, seja de micronutrientes ou desnutrição crônica 46 . Além disso, foi verificado que as medidas de associação incluindo e excluindo esses adolescentes não apresentaram diferenças significativas. Como pontos fortes, podemos citar o grande tamanho e abrangência amostral, com representatividade nacional e regional.

A associação entre refeições com os pais/responsáveis e obesidade necessita de maiores investigações no contexto brasileiro e a avaliação de outras variáveis relacionadas à refeição em família como: tipo de alimento consumido e a disponibilidade de ambiente familiar doméstico tranquilo e harmonioso; tempo, planejamento e organização das refeições; a divisão das atividades relacionadas ao preparo e o envolvimento dos jovens; e o local onde são feitas estas refeições. É preciso investigar as relações entre esses fatores do ambiente familiar doméstico e as refeições em família e como, juntas, influenciam o IMC de adolescentes.

Estudos futuros devem considerar não apenas o ambiente familiar, mas também as influências mais distais como da comunidade, cultural, geográfica, recursos socioeconômicos e políticas públicas nas esferas estadual e federal do país 47 . Cabe destacar que uma prática tão singela como refeição em família também é parte de um contexto econômico, social e cultural maior 33.

\section{Conclusão}

Os adolescentes do sexo masculino e mais novos estão mais protegidos da obesidade ao compartilharem o almoço e o jantar com os pais/responsáveis. Os resultados reforçam a importância da promoção de comportamentos alimentares saudáveis no ambiente familiar, contribuindo para a prevenção da obesidade nos adolescentes brasileiros. A refeição com os pais/responsáveis deve ser incentivada, pois pode fornecer oportunidade para uma alimentação adequada, com melhoria da disponibilidade e acessibilidade de alimentos saudáveis e bem-estar psicossocial dos adolescentes. 


\section{Colaboradores}

J. I. Silva contribuiu na análise e interpretação dos dados, elaboração, redação e revisão do artigo. A. C. $S$. Andrade contribuiu na análise e interpretação dos dados, revisão da versão final do artigo. K. V. Bloch contribuiu concepção e planejamento do estudo, análise e interpretação dos dados, revisão da versão final do artigo. G. S. Brunken contribuiu concepção e planejamento do estudo, análise e interpretação dos dados, elaboração e revisão do artigo.

\section{Informações adicionais}

ORCID: Juliana Ilídio da Silva (0000-0002-06046662); Amanda Cristina de Souza Andrade (00000002-3366-4423); Katia Vergetti Bloch (00000002-6992-3159); Gisela Soares Brunken (00000001-9626-4980).

\section{Agradecimentos}

À Coordenação de Aperfeiçoamento de Pessoal de Nível Superior (CAPES). Programa de Pós-graduação em Saúde Coletiva da Universidade Federal de Mato Grosso.

\section{Referências}

1. NCD Risk Factor Collaboration (RCD-RisC). Worldwide trends in body-mass index, underweight, overweight, and obesity from 1975 to 2016: a pooled analysis of 2416 populationbased measurement studies in 128,9 million children, adolescents, and adults. Lancet 2017; 390:2627-42.

2. Hruby A, Hu FB. The epidemiology of obesity: a big picture. Pharmacoeconomics 2015; 33:673-89.

3. Instituto Brasileiro de Geografia e Estatística. Pesquisa de Orçamentos Familiares, 20082009: antropometria e estado nutricional de crianças, adolescentes e adultos do Brasil. Rio de Janeiro: Instituto Brasileiro de Geografia e Estatística; 2010.

4. Fulkerson JA, Larson N, Horning M, Neumark-Sztainer D. A review of associations between family or shared meal frequency and dietary and weight status outcomes across the lifespan. J Nutr Educ Behav 2014; 46:2-19.

5. Martin-Biggers J, Spaccarotella K, BerhauptGlickstein A, Hongu N, Worobey J, ByrdBredbenner C. Come and get it! A discussion of family mealtime literature and factors affecting obesity risk. Adv Nutr 2014; 5:235-47.

6. Barlow SE; Expert Committee. Expert Committee recommendations regarding the prevention, assessment, and treatment of child and adolescent overweight and obesity: summary report. Pediatrics 2007; 120 Suppl 4:s163-s92.

7. Berge JM, Arikian A, Doherty WJ, NeumarkSztaine D. Healthful eating and physical activity in the home environment: results from multi-family focus groups. J Nutr Educ Behav 2012; 44:123-31.

8. Reid M, Worsley A, Mavondo F. The obesogenic household: factors influencing dietary gatekeeper satisfaction with family diet. Psychol Mark 2015; 32:544-57.

9. Neumark-Sztainer D, Larson NI, Fulkerson JA, Eisenberg ME, Story M. Family meals and adolescents: what have we learned from Project EAT (Eating Among Teens)? Public Health Nutr 2010; 13:1113-21.

10. Dallacker M, Hertwig R, Mata J. The frequency of family meals and nutritional health in children: a meta-analysis. Obes Rev 2018; 19:63853.

11. Valdés J, Rodríguez-Artalejo F, Aguilar L, Jaén-Casquero MB, Royo-Bordonada MA. Frequency of family meals and childhood overweight: a systematic review. Pediatr Obes 2012; 8:e1-e13.

12. Vasconcellos MTL, Silva PLN, Szklo M, Kuschnir MCC, Klein CH, Abreu GA, et al. Desenho da amostra do Estudo do Risco Cardiovascular em Adolescentes (ERICA). Cad Saúde Pública 2015; 31:921-30.

13. Silva TLN, Klein CH, Souza AM, Barufaldi LA, Abreu GA, Kuschnir MCC, et al. Participação no Estudo de Riscos Cardiovasculares em Adolescentes - ERICA. Rev Saúde Pública 2016; 50 Suppl 1:3s. 
14. Bloch KV, Szklo M, Kuschnir MCC, Abreu GA, Barufaldi LA, Klein CH, et al. The study of cardiovascular risk in adolescents - ERICA: rationale, design and sample characteristics of a national survey examining cardiovascular risk factor profile in Brazilian adolescents. BMC Public Health 2015; 15:94.

15. Onis M, Onyango AW, Borghi E, Siyam A, Nishida C, Siekmann J. Development of a WHO growth reference for school-aged children and adolescents. Bull World Health Organ 2007; 85:660-7.

16. World Health Organization. Global recommendations on physical activity for health. Geneva: World Health Organization; 2010.

17. Levy RB, Castro IRR, Cardoso LO, Tavares LF, Sardinha LMV, Gomes FS, et al. Consumo e comportamento alimentar entre adolescentes brasileiros: Pesquisa Nacional de Saúde do Escolar (PeNSE), 2009. Ciênc Saúde Colet 2010; 15 Suppl 2:3085-97.

18. Barufaldi LA, Abreu GA, Veiga GV, Sichieri R, Kuschnir MCC, Cunha DB, et al. Programa para registro de recordatório alimentar de 24 horas: aplicação no Estudo de Riscos Cardiovasculares em Adolescentes. Rev Bras Epidemiol 2016; 19:464-8.

19. Institute of Medicine. Dietary, functional, and total fiber. In: Institute of Medicine, editor. Dietary reference intakes for Energy, carbohydrate, fiber, fat, fatty acids, cholesterol, protein, and amino acids (macronutrients). https:// www.nal.usda.gov/sites/default/files/fnic_up loads/energy_full_report.pdf (acessado em 19/ Mar/2017).

20. Parikh S, Pollock NK, Bhagatwala J, Guo D-H, Gutin B, Zhu H, et al. Adolescent fiber consumption is associated with visceral fat and inflammatory markers. J Clin Endocrinol Metab 2012; 97:e1451-7.

21. Du H, van der A DL, Boshuizen HC, Forouhi NG, Wareham NJ, Halkjær J, et al. Dietary fiber and subsequent changes in body weight and waist circumference in european men and women. Am J Clin Nutr 2010; 91:329-36.

22. Instituto Brasileiro de Geografia e Estatística. Pesquisa Nacional de Saúde do Escolar, 2015. Rio de Janeiro: Instituto Brasileiro de Geografia e Estatística; 2016.

23. Martins BG, Ricardo CZ, Machado PP, Rauber F, Azeredo CM, Levy RB. Fazer refeições com os pais está associado à maior qualidade da alimentação de adolescentes brasileiros. Cad Saúde Pública 2019; 35:e00153918.

24. Flament MF, Hill EM, Buchholzc A, Hendersonc K, Tasca GA, Goldfield G. Internalization of the thin and muscular body ideal and disordered eating in adolescence: the mediation effects of body esteem. Body Image 2012; 9:6875.

25. Neumark-Sztainer D. Preventing obesity and eating disorders in adolescents: what can health care providers do? J Adolesc Health 2009; 44:206-13.
26. Harrison ME, Norris ML, Obeid N, Fu M, Weinstangel H, Sampson M. Systematic review of the effects of family meal frequency on psychosocial outcomes in youth. Can Fam Physician 2015; 61:e96-106.

27. Goldfield GS, Murray MA, Buchholz A, Henderson K, Obeid N, Kukaswadia A, et al. Family meals and body mass index among adolescents: effects of gender. Appl Physiol Nutr Metab 2011; 36:539-46.

28. Haines J, Kleinman KP, Rifas-Shiman SL, Field AE, Austin SB. Examination of shared risk and protective factors for overweight and disordered eating among adolescents. Arch Pediatr Adolesc Med 2010; 164:336-43.

29. Larson NI, Wall MM, Story MT, NeumarkSztainer DR. Home/family, peer, school, and neighborhood correlates of obesity in adolescent. Obesity (Silver Spring) 2013; 21:1858-69.

30. Berge JM, Wall M, Larson N, Forsyth A, Bauer KW, Neumark-Sztainer D. Youth dietary intake and weight status: healthful neighborhood food environments enhance the protective role of supportive family home environments. Health Place 2014; 26:69-77.

31. Taveras EM, Rifas-Shiman SL, Berkey CS, Rockett HRH, Field AE, Frazier AL, et al. Family dinner and adolescent overweight. Obes Res 2005; 13:900-6.

32. Neumark-Sztainer DR, Wall MM, Haines JI, Story MT, Sherwood NE, van den Berg PA. Shared risk and protective factors for overweight and disordered eating in adolescents. Am J Prev Med 2007; 33:359-69.

33. Fiese BH, Hammons A, Grigsby-Toussaint D. Family mealtimes: a contextual approach to understanding childhood obesity. Econ Hum Biol 2012; 10:365-74.

34. Berge JM, Jin SW, Hannan P, Neumark-Sztainer D. Structural and interpersonal characteristics of family meals: associations with adolescent body mass index and dietary patterns. J Acad Nut Diet 2013; 113:816-22.

35. Oliveira JS, Barufaldi LA, Abreu GA, Leal VS, Brunken GS, Vasconcelos SML, et al. ERICA: uso de telas e consumo de refeições e petiscos por adolescentes brasileiros. Rev Saúde Pública 2016; 50 Suppl 1:7s.

36. Souza AM, Barufaldi LA, Abreu GA, Giannini DT, Oliveira CL, Santos MM, et al. ERICA: ingestão de macro e micronutrientes em adolescentes brasileiros. Rev Saúde Pública 2016; 50 Suppl 1:5s.

37. Fulkerson JA, Neumark-Sztainer D, Hannan PJ, Story M. Family meal frequency and weight status among adolescents: cross-sectional and 5-year longitudinal associations. Obesity (Silver Spring) 2008; 16:2529-34.

38. Dwyer L, Oh A, Patrick H, Hennessy E. Promoting family meals: a review of existing interventions and opportunities for future research. Adolesc Health Med Ther 2015; 6:11531 . 
39. Barufaldi LA, Abreu GA, Oliveira JS, Santos DF, Fujimori E, Vasconcelos SML, et al. ERICA: prevalência de comportamentos alimentares saudáveis em adolescentes brasileiros. Rev Saúde Pública 2016; 50 Suppl 1:6s.

40. Birch L, Savage JS, Ventura A. Influences on the development of children's eating behaviours: from infancy to adolescence. Can J Diet Pract Res 2007; 68:s1-56.

41. Fundo Nacional de Desenvolvimento da Educação. Resolução no 26, de 17 de junho de 2013. Dispõe sobre o atendimento da alimentação escolar aos alunos da educação básica no âmbito do Programa Nacional de Alimentação Escolar - PNAE. Diário Oficial da União 2013; 18 jun.

42. Carmo AS, Assis MM, Cunha CF, Oliveira TRPR, Mendes LL. The food environment of Brazilian public and private schools. Cad Saúde Pública 2018; 34:e00014918.

43. Fulkerson JA, Pasch KE, Stigler MH, Farbakhsh K, Perry CL, Komro KA. Longitudinal associations between family dinner and adolescente perceptions of parent-child communication among raciallydiverse urban youth. J Fam Psychol 2010; 24:261-70.
44. Larson NI, Neumark-Sztainer D, Hannan PJ, Story M. Family Meals during adolescence are associated with higher diet quality and healthful meal patterns during young adulthood. J Am Diet Assoc 2007; 107:1502-10.

45. Berge JM, Wall M, Hsueh T-F, Fulkerson JA, Larson N, Neumark-Sztainer D. The protective role of family meals for youth obesity: 10-year longitudinal associations. J Pediatr 2015; 166:296-301.

46. Departamento de Atenção Básica, Secretaria de Atenção à Saúde, Ministério da Saúde. Guia alimentar para a população brasileira. 2a Ed. Brasília: Ministério da Saúde; 2014.

47. Harrison K, Bost KK, McBride BA, Donovan SM, Grigsby-Toussaint DS, Kim J, et al. Toward a developmental conceptualization of contributors to overweight and obesity in childhood: the six-Cs model. Child Dev Perspect $2011 ; 5: 50-8$. 


\section{Abstract}

The study aimed to analyze the association between sharing lunch or supper with parents/ guardians and obesity in Brazilian adolescents participating in the Study of Cardiovascular Risk Factors in Adolescents (ERICA in Portuguese). This was a cross-sectional, school-based study with adolescents 12 to 17 years of age. Obesity was classified by body mass index based on World Health Organization criteria, according to age and sex. The association between obesity and sharing lunch and supper with parents/ guardians (never, sometimes, almost every day, and every day) was analyzed according to crude and sex- and age-adjusted prevalence ratios. A total of 71,740 adolescents were assessed. Of these, $48 \%$ and $60 \%$ of girls and $56 \%$ and $65 \%$ of boys, respectively, shared lunch and supper every day or nearly every day. Boys who shared lunch and supper with their parents/guardians nearly every day and every day showed lower prevalence of obesity. Stratified by age bracket, only younger boys that ate lunch with their parents/guardians sometimes $(P R=0.64 ; 95 \% C I: 0.46-0.89)$, nearly every day $(P R=0.50 ; 95 \% C I: 0.37-0.69)$, and every day (PR $=0.65 ; 95 \%$ CI: 0.49-0.85) and supper with their parents/guardians every day ( $P R=0.61 ; 95 \% C I$ : 0.43-0.87) showed lower prevalence of obesity. The findings highlight the importance of promoting healthy eating behaviors in the family setting as part of strategies to prevent obesity in adolescents.

Adolescent; Obesity; Body Mass Index; Meals; Health Surveys

\section{Resumen}

El objetivo del estudio fue analizar la asociación de la frecuencia de comer y cenar con los padres/ tutores y la obesidad en adolescentes brasileños participantes del Estudio de Riesgos Cardiovasculares en Adolescentes (ERICA). Se trata de un estudio seccional, con base escolar, en adolescentes de 12 a 17 años. La obesidad fue clasificada por el índice de masa corporal, basado en los criterios de la Organización Mundial de la Salud, según edad y sexo. La asociación entre obesidad, comida y cena con los padres/tutores (nunca, a veces, casi todos los días y todos los días) se investigó por la razón de prevalencia bruta y ajustada para estratos de sexo y franja de edad. Se evaluaron a 71.740 adolescentes. De esos, comían y cenaban con los padres/responsables todos los días o casi todos los días, respectivamente, cerca de un $48 \%$ y un 60\% de las niñas y un 56\% y 65\% de los niños. Los niños que comían y cenaban con los padres/ responsables casi todos los días y todos los días presentaron menor prevalencia de obesidad. En la estratificación por franja de edad, solamente los niños más jóvenes que afirmaron comer con los padres/responsables a veces $(R P=0,64$; IC95\%: 0,46-0,89), casi todos los días $(R P=0,50$; IC95\%: $0,37-0,69)$ y todos los días $(R P=0,65$; IC95\%: $0,49-0,85)$ y cenar con los padres/responsables todos los días ( $R P=0,61$; IC95\%: 0,43-0,87) presentaron menor prevalencia de obesidad. Los resultados refuerzan la importancia de la promoción de comportamientos alimentarios saludables en el ambiente familiar como parte de las estrategias de prevención de la obesidad en adolescentes.

Adolescente; Obesidad; Índice de Masa Corporal; Comidas; Encuestas Epidemológicas
Recebido em 03/Jun/2019

Versão final reapresentada em 02/Dez/2019

Aprovado em 15/Jan/2020 